Archives

18-19 | 1997

Inventaire des archives de Robert Mandrou

\title{
Il y a tout juste trente ans...
}

\section{Henriette Asséo}

\section{(2) OpenEdition Journals}

\section{Édition électronique}

URL : http://journals.openedition.org/ccrh/2559

DOI : $10.4000 /$ ccrh.2559

ISSN : $1760-7906$

Éditeur

Centre de recherches historiques - EHESS

Édition imprimée

Date de publication : 10 octobre 1997

ISSN : 0990-9141

Référence électronique

Henriette Asséo, « II y a tout juste trente ans... », Les Cahiers du Centre de Recherches Historiques [En ligne], 18-19 | 1997, mis en ligne le 20 février 2009, consulté le 20 avril 2019. URL : http:// journals.openedition.org/ccrh/2559; DOI : 10.4000/ccrh.2559

Ce document a été généré automatiquement le 20 avril 2019

Article L.111-1 du Code de la propriété intellectuelle. 


\title{
Il y a tout juste trente ans...
}

\author{
Henriette Asséo
}

1 Chaque jeudi matin, Robert Mandrou gagnait d'un pas ferme le grand amphithéâtre situé dans le hall central de la faculté de Nanterre pour y prodiguer un cours de licence. Tout dans le style de notre professeur contrastait avec l'atmosphère nerveuse de cette rentrée d'octobre 1968: une stature assez mandarinale, des dossiers couverts d'une écriture élégante ; une tenue élégante en accord avec la couleur de ses yeux d'un bleu très clair. La faune étudiante arpentait les mêmes couloirs. On a conféré ultérieurement à cette génération une réputation bien surfaite d'excentricité. Les documents de l'époque montrent des jeune gens imberbes et des jeunes filles aux jupes tout juste un peu courtes. Tout changeait pourtant quand on écoutait ce qui se clamait quotidiennement dans le hall central fort mal éclairé. Aussi pâteux que l'épaisse couche de fumée collée en permanence au plafond, un verbe surréel flottait, amplifié par des mégaphones mal réglés, diffusant tour à tour, ou simultanément, la bonne parole trotskiste, maoïste, chrétienne ou anarchiste. L'atmosphère était à la mobilisation permanente et des militants dotés d'un esprit de vigilance éminemment "prolétarienne ", traquaient des ennemis (les fascistes et les CRS) aussi abhorrés qu'invisibles. Ils n'accordaient qu'un regard de mépris aux étudiants accomplissant l'acte d'assister à un cours magistral (quel qu'en fût le contenu intellectuel). S'ils nous traitaient de collaborateurs et de traîtres, nous les considérions volontiers comme des fascistes et des hystériques. Nous n'aurions pour rien au monde avoué qu'il existait entre eux et nous une complicité tacite: le cynisme partagé par les enfants sages d'après-guerre.

2 Car la génération dite de « Mai 68 » était entrée en faculté avec des dispositions d'esprit semblables, par-delà la variété des conditions sociales et familiales. L'enseignement y avait été inauguré en 1964 par une propédeutique à peine plus encombrée d'étudiants que les classes de lycée. Dans un premier temps, l'ordinaire des espérances de la jeunesse d'alors fut grandement amélioré par une mixité toute neuve. Approcher l'autre sexe justifiait la fréquentation de la bibliothèque, faute de café à la périphérie. On se mit bientôt à l'heure du couscous sans trop s'affliger du spectacle des baraquements du bidonville tout proche. 
3 Puis, très vite, le vide aspira à nouveau les étudiants. Ils ne surent énoncer leurs désarrois et les pensèrent de la couleur du temps. Ainsi va le monde, croyaient-ils : une insertion sociale moyenne, des métiers sans mystère, mariages et divorces, enfants bien reçus et mal élevés remplaceraient le destin dramatique des parents. C'est alors que certains d'entre eux décidèrent de prendre pied en terre étrangère. Ils ne savaient rien de l'Arabe et de sa langue, du Vietnamien et du napalm, rien de l'Indien ni du cuivre. Cette génération militante était dotée, par un reste d'imagination enfantine, d'une double culpabilité : celle d'être née après la guerre, celle de n'être d'aucun combat. Elle se mit à adorer un Arabe méconnu, à aduler un Africain à peine côtoyé, à chanter un LatinoAméricain écartelé mais infréquentable. Elle se jugea importante et fut seulement importune. Petites misères, petites cellules, un marxisme de bazar, les hasards de la dialectique. Être ou ne pas être au Parti? Le défi d'Hamlet avait été posé en reprise de carte.

4 Puis, elle prit goût pour la théorie. Elle justifia à toutes les sauces sa volonté de certitudes, prit les loups pour les chiens et le dénigrement pour la praxis. Les militants n'étaient jamais pris au dépourvu dans le débat public (10 personnes au maximum), dans le dialogue avec les masses (20 tout au plus), dans l'abattage à la Roneo du tract provocant et bien mal rédigé. Ils se groupaient en formations serrées, se croisaient dans la salle de l'UNEF sans se parler. Ils engageaient des flirts théoriques passionnés, bientôt suivis de mariages politiques raisonnables, puis de querelles intestines et d'anathèmes fulgurants. Vingt, puis dix, puis cinq... selon une arithmétique singulière, chaque éclatement leur paraissait triomphant. Peu nombreux sans doute, mais plus près des masses. D'ailleurs, ils cherchaient moins à être convaincus, qu'à convaincre. Guère généreux, ils vouaient aux gémonies toute marque de compassion et brisaient leurs tendresses désertées dans des pudeurs glacées. Ils parlaient souvent d'eux-mêmes, jamais des leurs. Ils se rencontraient en terrain neutre, la Cité universitaire, matrice froide aux couloirs d'hôpital.

5 Je ne comprends toujours pas comment un psychodrame à usage interne a pu se muer en psychodrame national. J'ai longtemps eu le sentiment d'appartenir à un monde de plagiaires, de pâles copies en perpétuel devenir de nos parents aimés. Génération de l'impossible révolution, nous avons bien vite eu honte de notre savoir-faire. Élèves modèles, nous avons tout fait pour rater nos examens et conquérir ainsi le droit à la vie. Ceux qui rêvaient de vivre dangereusement, militent pour le K 12 de la Sécurité sociale, selon une expression qui était chère à Robert Mandrou ; certains ont troqué le verbe pour le portefeuille. Déjà il y avait eu, chez tant de bons jeunes gens (et jeunes filles) de cette génération, un systématique détournement de la réalité qui tenait du prodige.

6 Pourtant c'était hier, semble-t-il, que nous accompagnions notre professeur avec nos classeurs sous le bras. Mais on n'entre pas dans la vie à reculons et le témoignage rêvé ne suffira pas à assurer notre rédemption.

7 Pour quelles raisons Robert Mandrou éprouva-t-il, pendant ces années de professeur à Nanterre une véritable jubilation à se confronter presque quotidiennement à cette ambiance péremptoire et ouvertement anti-intellectuelle ? S'agissait-il d'un combat pour l'histoire qui redonnait sens à l'acte d'enseigner? Je crois qu'il y avait autre chose qui est de l'ordre impalpable des conjonctures mentales. En d'autres termes, un alliage instable, sans cause véritable et sans objet défini, entre les générations, l'espace d'une saison... ainsi, en d'autres temps, Jules Michelet, Victor Cousin. 
8 Robert Mandrou n'a pas prodigué un enseignement de combat pas plus qu'un enseignement dit « critique ", selon la formule consacrée à l'époque. Par son exigence de rigueur et la limpidité de son style, il nous fit simplement comprendre la nécessité de « la lutte qui fait reculer la délation et l'hallucination ", ainsi qu'il l'écrivit à propos de Michelet en 1964, dans la préface à se réédition de La Sorcière. Exigence à mener d'abord pour soi-même.

9 Je crois pouvoir dire que nous sommes un certain nombre, parmi ses étudiants, à être définitivement marqués par la formation qu'il nous a donnée. Et comment en serait-il autrement puisqu'il nous a réconciliés avec notre pays en authentifiant un sentiment national longtemps désavoué ! Et comment en serait-il autrement puisqu'il nous conduisit vers une Allemagne qui n'avait plus rien à voir avec la maison mortuaire de certains cauchemars d'enfance! Et comment en serait-il autrement puisqu'il nous fit connaître l'autre foyer des Humanistes aux hommes de science! La culture profonde de l'Europe centrale - de la Pologne, de la Hongrie ou de la Bohème - était encore largement, à l'époque, tenue en mésestime. Comme le constatait Czeslaw Milosz, dès 1959 dans Une autre Europe, elle ne passait guère le mur de l'indifférence d'intellectuels soucieux d'oppressions plus lointaines.

10 Robert Mandrou eût aimé voir l'Europe d'aujourd'hui, telle qu'elle est, à nouveau au cœur de l'Histoire, pour le meilleur et pour le pire. 